\title{
Amygdala-Hippocampal Connectivity Changes During Acute Psychosocial Stress: Joint Effect of Early Life Stress and Oxytocin
}

\author{
Yan Fan*,1,2,3, Karin Pestke', Melanie Feeser ${ }^{1,2,3}$, Sabine Aust ${ }^{1,2,3}$, Jens C Pruessner ${ }^{4,5}$, Heinz Böker ${ }^{6}$, \\ Malek Bajbouj ${ }^{1,2,3,7}$ and Simone Grimm ${ }^{1,2,3,6,7}$ \\ 'Cluster of Excellence 'Languages of Emotion', Freie Universität Berlin, Berlin, Germany; 'Department of Psychiatry, Campus Benjamin Franklin, \\ Charité Berlin, Berlin, Germany; ${ }^{3}$ Dahlem Institute for Neuroimaging of Emotion, Freie Universität Berlin, Berlin, Germany; ${ }^{4}$ Douglas Mental Health \\ Institute, Department of Psychiatry, McGill University, Montreal, Canada; ${ }^{5}$ Montreal Neurological Institute, McGill University, Montreal, Canada; \\ ${ }^{6}$ Department of Psychiatry, Psychotherapy and Psychosomatics, Hospital of Psychiatry, University of Zurich, Zurich, Switzerland
}

Previous evidence shows that acute stress changes both amygdala activity and its connectivity with a distributed brain network. Early life stress (ELS), especially emotional abuse (EA), is associated with altered reactivity to psychosocial stress in adulthood and moderates or even reverses the stress-attenuating effect of oxytocin (OXT). The neural underpinnings of the interaction between ELS and OXT remain unclear, though. Therefore, we here investigate the joint effect of ELS and OXT on transient changes in amygdala-centered functional connectivity induced by acute psychosocial stress, using a double-blind, randomized, placebo-controlled, within-subject crossover design. Psychophysiological interaction analysis in the placebo session revealed stress-induced increases in functional connectivity between amygdala and medial prefrontal cortex, posterior cingulate cortex, putamen, caudate and thalamus. Regression analysis showed that EA was positively associated with stress-induced changes in connectivity between amygdala and hippocampus. Moreover, hierarchical linear regression showed that this positive association between EA and stress-induced amygdala-hippocampal connectivity was moderated after the administration of intranasal OXT. Amygdala-hippocampal connectivity in the OXT session correlated negatively with cortisol stress responses. Our findings suggest that altered amygdala-hippocampal functional connectivity during psychosocial stress may have a crucial role in the altered sensitivity to OXT effects in individuals who have experienced EA in their childhood.

Neuropsychopharmacology (20I5) 40, 2736-2744; doi:I 0.I038/npp.20I 5.I23; published online 27 May 2015

\section{INTRODUCTION}

Appropriate neural and physiological responses to transient shifts of homeostasis during stress are crucial for our survival and well-being (McEwen, 2003). The deterioration of homeostasis renders the individual vulnerable to the cumulative burden of stress. Cumulative stress early in life comprises a spectrum of adverse social experiences that occur before puberty, including sexual, physical, and emotional forms of abuse, as well as physical and emotional neglect (Pechtel and Pizzagalli, 2011). Ample evidence supports that early life stress (ELS) has enduring impact on the neuroendocrine system and leads to dysregulated cortisol responses to psychosocial stress in adulthood (Strüber et al,

*Correspondence: Y Fan, Klinik für Psychiatrie und Psychotherapie, Charité-Universitätsmedizin Berlin, Campus Benjamin Franklin, Hindenburgdamm 30, Berlin 12203, Germany, Tel: +49 (0)30 450 517778, Fax: +49 (0)30 450 517942, E-mail: yan.fan@fu-berlin.de or yan.fan@charite.de

7 These authors contributed equally to this work.

Received 7 October 2014; revised I5 March 2015; accepted 6 April 2015; accepted article preview online 29 April 2015
2014). Such ELS-associated changes in stress reactivity have been associated with the development of depressive and anxiety disorders later in life (McEwen, 2003). However, the neural mechanisms underlying the impact of ELS on stress reactivity have not yet been elucidated.

Stress reactivity is regulated in the central nervous system through the coordinated circuitry coupling among limbic and prefrontal brain regions, such as the amygdala, hippocampus, and medial prefrontal cortex (mPFC) (Herman et al, 2005). As a key locus in the limbic-prefrontal circuit, the amygdala is reciprocally interconnected with regions involved in autonomic-neuroendocrine control, memory, and salience processing (Freese and Amaral, 2009). Amygdala activity and connectivity signal the emotional significance of the perceived stimuli and prepare our brain and body for suitable responses (LeDoux, 2000). Changes in functional connectivity (FC) between amygdala and other limbic-prefrontal regions have been demonstrated during and after stress induction (Hermans et al, 2011; Vaisvaser et al, 2013; Veer et al, 2011). Particularly, enhanced amygdala-hippocampal FC has been found both in the immediate responses to and in the prolonged aftermath of 
stress (Vaisvaser et al, 2013). The hippocampus inhibits the hypothalamic-pituitary-adrenal (HPA) axis and receives negative feedback from its hormonal end product cortisol (Herman et al, 2005). Amygdala-hippocampal FC is inversely associated with cortisol levels (Vaisvaser et al, 2013) and predicts the capacity of the HPA axis to restore homeostasis after perturbation (Kiem et al, 2013). These previous findings demonstrate that amygdala-hippocampal FC has a critical role in responses to transient stress, especially for the restoration and maintenance of homeostasis via neuroendocrine control. It remains unclear though whether not only transient but also sustained/cumulative stress early in life is associated with altered amygdala-hippocampal FC.

ELS is related to changes in gray and white matter in stress regulation regions, such as amygdala, hippocampus, and mPFC. ELS-associated deficits in structural connectivity among these regions are also evident, supporting network abnormalities in the limbic-prefrontal circuit (Hart and Rubia, 2012). Recent studies have found ELS-associated decreases in resting-state FC (rs-FC) within the limbicprefrontal circuit (Burghy et al, 2012; Fan et al, 2014; Herringa et al, 2013). Although rs-FC is postulated to reflect trait-like individual differences in circuitry integrity, taskevoked FC changes (eg, measured by psychophysiological interactions (PPIs)) are supposed to reflect transient changes in brain network coupling in response to specific task demands. Gee et al (2013) reported changes in amygdalamPFC FC during emotion perception in adolescents who have experienced ELS. In line with the recent discovery of stress-dependent epigenetic changes in the hippocampus (Klengel et al, 2013), Holz et al (2015) investigated subjects with a genetic variant that makes them more sensitive to stress-dependent epigenetic modifications in the hippocampus and reported enhanced amygdala-hippocampus FC during emotion perception in these individuals. These findings further support that task-evoked FC changes in the limbic-prefrontal circuit are also influenced by ELS. For this reason, stress-induced changes in amygdala-centered FC are of primary importance for understanding the impact of ELS on stress reactivity. However, so far no study has investigated the influence of ELS on the transient FC shift within the limbic-prefrontal circuit during acute psychosocial stress.

Furthermore, ELS has long-lasting effects on the development of the oxytocinergic system, in which the secretion of oxytocin (OXT) inhibits both basal and stress-induced HPA axis activity (Neumann et al, 2000). Intranasal administration of OXT attenuates cortisol stress responses. However, in subjects who have experienced ELS, this stress-attenuating effect of OXT is diminished or even reversed (Grimm et al, 2014). The neural substrates involved in the altered sensitivity to OXT after ELS remain unclear. ELS is associated with decreased cerebrospinal fluid (CSF) OXT level in adulthood (Heim et al, 2009). Rodent models show that ELS reduces the expression of OXT receptors in the amygdala (Hill et al, 2014). Recent studies on fear and salience arousal show that OXT reduces amygdala activation and alters amygdala-centered FC to brain regions involved in autonomic-neuroendocrine control (Riem et al, 2012). In our recent study, contrary to the stress-attenuating effect in control subjects, OXT enhanced cortisol stress responses and stress-induced deactivation in limbic areas in subjects who experienced ELS (Grimm et al, 2014). It remains unclear whether stress-induced changes in amygdala-centered FC are also modulated by the joint effect of OXT and ELS.

The primary aim of the present study was to investigate the effect of ELS on stress-induced changes in amygdalacentered FC. Task-evoked FC changes between amygdala and other limbic-prefrontal regions have been demonstrated during transient acute stress (Hermans et al, 2011; Vaisvaser et al, 2013; Veer et al, 2011). It has also been shown that ELS impacts structural connectivity and trait-like rs-FC within the limbic-prefrontal circuit (Burghy et al, 2012; Fan et al, 2014; Hart and Rubia, 2012; Herringa et al, 2013). Here we hypothesize that ELS influences stress-induced FC changes from amygdala to $\mathrm{mPFC}$ and hippocampus. Our second aim was to explore the interaction between ELS and OXT on stress-induced amygdala FC shift. Grimm et al (2014) found that the hippocampus is involved in the interaction effects of ELS and OXT on stress reactivity. We therefore hypothesize a joint effect of EA and OXT on stress-induced FC changes between amygdala and hippocampus.

\section{MATERIALS AND METHODS}

\section{Assessment of ELS}

ELS was measured using the Childhood Trauma Questionnaire (CTQ; Bernstein and Fink, 1998). The CTQ is a 28-item self-report questionnaire that retrospectively assesses five types of adverse childhood experiences: emotional neglect $(\mathrm{EN})$, emotional abuse (EA), physical neglect $(\mathrm{PN})$, physical abuse (PA), and sexual abuse (SA). Scores range from 5 to 25 for each subscale, with high scores indicating strong exposure to the corresponding type of maltreatment.

It has been found that different subtypes of ELS have differential impact on stress reactivity and stress-related affective disorders (Carpenter et al, 2009; Strüber et al, 2014). For example, unlike other subtypes of ELS, EA is a particularly strong predictor for blunt HPA reactivity, dampened cortisol stress responses, and internalizing psychopathology, such as major depression and social anxiety (Carpenter et al, 2009; Grimm et al, 2014; Keyes et al, 2012). Strong effects of EA have also been found on trait-like rs-FC within the limbic-prefrontal circuit, as well as on OXT concentrations in the CSF (Fan et al, 2014; Heim et al, 2009). For this reason, we focused on EA as a main predictor in the multiple regression analyses.

\section{Subjects}

Thirty-two healthy young male participants (aged 21-37 years, mean age $28.3 \pm 4.7)$ were recruited from a preexisting community-dwelling sample $(N=541$; Fuge et al, 2014). The short version of the Structured Clinical Interview for Diagnostic and Statistical Manual of Mental Disorders (Wittchen et al, 1997) was used to screen for history of psychiatric or neurological disease. Verbal intelligence was assessed using a German word recognition test (Lehrl et al, 1995; Schmidt and Metzler, 1992), which is functionally equivalent to the widely used National Adult Reading Test (Nelson and O'Connell, 1978). Inclusion criteria were age 18-40 years, absence of present and past diagnosis of psychiatric or neurologic disease, absence of major or unstable general medical conditions, IQ score (verbal intelligence) 
$>90$, and ability to participate in MRI study procedures. This study belongs to a project probing OXT effects on resting-state network (Fan et al, 2014) and stress reactivity (Grimm et al, 2014). The study was conducted in accordance with the latest version of the Declaration of Helsinki and approved by the Institutional Review Board of the Germany Psychological Society. All subjects gave written informed consent before screening and were reimbursed for participation.

\section{Study Design}

In a double-blind, placebo-controlled, within-subject crossover design, participants received either OXT (Syntocinon Spray; Novartis, Basel, Switzerland) or a placebo (PLA; sodium chloride solution) intranasally in two separate experimental sessions. The sequence of OXT and PLA sessions was counter-balanced across subjects. We adopted a standardized protocol used in previous studies (Gamer et al, 2010; Labuschagne et al, 2010) where participants selfadministered a single intranasal dose of 24 IU OXT/PLA (three puffs per nostril, each puff with $4 \mathrm{IU}$, or $6.82 \mathrm{mg}$ ) under the supervision of the experimenter $45 \mathrm{~min}$ before each fMRI scanning session. Participants abstained from alcohol during the $24 \mathrm{~h}$ and from exercise, caffeine, and food for $1 \mathrm{~h}$ before the experiment.

\section{Induction of Psychosocial Stress}

In each scanning session, psychosocial stress was induced using the Montreal Imaging Stress Task (MIST; Pruessner et al, 2008), in which the subjects completed a series of challenging mental arithmetic tasks. The MIST utilizes a block design and consists of three runs (each lasts for $7 \mathrm{~min}$ ). Within each run, three conditions (ie, $0.5 \mathrm{~min}$ rest, $1 \mathrm{~min}$ control, and 2 min stress) were presented in this order and repeated once. In contrast to the control condition, the difficulty and time limit of the mental arithmetic tasks were manipulated in the stress condition, and the subjects were given negative feedbacks. Psychosocial stress was hence induced via elements of uncontrollability and social evaluative threat (for full description of the MIST, see Supplementary Methods, as well as Grimm et al, 2014).

\section{Psychological and Physiological Measures}

Before scanning started, the subjects completed the German versions of the Spielberger State-Trait Anxiety Inventory (STAI; Spielberger and Sydeman, 1994), the NEO five-factor inventory (Costa and McCrae, 1985), and the Rosenberg Self-Esteem scale (Rosenberg, 1965). To validate the stress induction, feelings of negativity and uncontrollability were assessed immediately before and after the fMRI scanning sessions via the state part of the STAI (Spielberger and Sydeman, 1994). Saliva samples were collected with the Salivette sampling device (Sarstedt) before (ie, $45 \mathrm{~min}$ after OXT/PLA administration) and immediately after the MIST task (for full description, see Grimm et al, 2014). Salivaderived cortisol was analyzed using a time-resolved fluorescence immunoassay (Dressendorfer et al, 1992). The subjective report of state anxiety and salivary cortisol levels before and after stress induction were compared by paired- sample $T$-tests. Cortisol stress responses were calculated as the difference between salivary cortisol levels before and after psychosocial stress.

\section{MRI Acquisition}

Structural and functional MRI data were acquired on a Siemens Trio 3T scanner using a 12-channel radio-frequency (RF) head coil. T1-weighted structural images were acquired with the following parameters: 176 sagittal slices covering the whole brain, repetition time $(\mathrm{TR})=1900 \mathrm{~ms}$, echo time $(\mathrm{TE})=2.52 \mathrm{~ms}$, flip angle $=9^{\circ}, 256 \times 256$ matrix, voxel size $1 \times 1 \times 1 \mathrm{~mm}^{3}$. In the OXT and PLA sessions, respectively, 3 runs of 220 volumes of $\mathrm{T}^{\star}$-weighted echo-planar images were acquired with the following parameters: 37 axial slices of $3 \mathrm{~mm}$ thickness covering the whole brain, repetition time $(\mathrm{TR})=2000 \mathrm{~ms}$, echo time $(\mathrm{TE})=30 \mathrm{~ms}$, flip angle $=70^{\circ}$, $64 \times 64$ matrix, field of view $=192 \times 192 \mathrm{~mm}^{2}$, in-plane resolution $=3 \times 3 \mathrm{~mm}^{2}$.

\section{FMRI Preprocessing}

Functional images were preprocessed using MATLAB 2012 (The Mathworks, Natick, MA, USA) and SPM8 (Statistical parametric mapping software, SPM; Wellcome Department of Imaging Neuroscience, London, UK; http://www.fil.ion. ucl.ac.uk). The images were corrected for the acquisition time differences between slices, realigned to the first volume to correct for head motion between volumes, then normalized into standard stereotactic space (template provided by the Montreal Neurological Institute) using the normalization parameters estimated by $\mathrm{T} 1$ structural image unified segmentation, and finally smoothed with a Gaussian kernel of $8 \mathrm{~mm}$ full-width-half-maximum.

\section{FC Analysis: PPI}

Whole-brain-wise PPI analyses (Friston et al, 1997) were performed to examine how amygdala-centered FC pattern is modulated by acute psychosocial stress. The PPI analysis is a specific form of moderated multiple regression model. The predictors of this regression model include the time course of the experiment (psychological factor), the time course of a seed region (physiological factor), an interaction term of both, and covariates. Significant interaction effects between the psychological and physiological factors reveal how activity in a brain region covariates with activity in the seed region in response to the experimental manipulation.

We used the standard implementation of PPI in SPM8 and the generalized PPI toolbox (gPPI; http://www.nitrc.org/ projects/gppi) introduced by McLaren et al (2012), which allows including more than one experimental term in the multiple regression model. In this manner, the psychological factors, ie, three experimental conditions (rest, control, stress) of the MIST, are represented by three separate regressors in the PPI model. Generalized PPI hence measures how FC strength changes for each task condition relative to the implicit baseline (eg, fixation) and is shown to be more sensitive and accurate for assessing pair-wise connectivity differences between conditions (eg, stress $>$ control) when there are more than two conditions in the task. 
Two seed regions were drawn in the left and right amygdala according to the intersection between an anatomical mask (Automated Anatomical Labeling; TzourioMazoyer et al, 2002) and the result of a meta-analysis of 163 fMRI studies on emotional processing (Wager et al, 2012). For each participant and each seed region, the physiological factor is represented by the first eigenvariates of the BOLD signal within the seed region, which were highpass filtered with a cutoff of $128 \mathrm{~s}$, prewhitened to account for serial autocorrelations of order $1(\operatorname{AR}(1))$, adjusted for an F-contrast over all experimental conditions, and deconvolved using the canonical Haemodynamic Response Function. Interaction terms were calculated separately for each experimental condition as the product between the vector of the condition and the physiological factor. The resulting contrast image 'stress $v s$ control' reveals stress-induced FC change to the corresponding seed region.

\section{Statistical Analysis}

First, to identify changes in FC pattern during acute psychosocial stress, a voxel-wise one-sample $T$-test was performed as second-level analysis on the contrast 'stress $v s$ control' in the placebo session. To investigate the effect of EA on stress-induced FC changes in the placebo session, we performed a multiple regression analysis on the contrast image 'stress $v s$ control' with EA as the main predictor and controlled for the following three covariates: age, IQ, and experiment sequence. All regressors were included in one model. Mean-centered predictors were used to reduce multicollinearity. Results are reported at a threshold of cluster-level FWE-corrected $p<0.05$, cluster size $>50$ voxels.

In brain regions where a main effect of EA was found, we built regions of interest (ROIs) and extracted FC strength for the stress and control conditions as beta values from the PPI model (see Supplementary Methods). Stress-induced FC changes were calculated as the differences in FC strength between the stress and control conditions. Previous studies have associated ELS with reduced gray matter volume (GMV) in limbic areas (Teicher et al, 2012) and with personality traits, such as neuroticism, trait anxiety, and selfesteem (Grimm et al, 2014). We further explored the influence of GMV and personality traits on the main effect of EA, using ROI-based multiple regression model (main predictor: EA; covariates: age, IQ, experimental sequence, GMV in seed region, GMV in the corresponding ROI, neuroticism, trait anxiety, and self-esteem).

To examine whether OXT and its joint effect with EA also modulates stress-induced FC change in these regions, we extracted stress-induced FC changes for the placebo and OXT sessions separately and performed a series of hierarchical multiple regression (HMR) analyses. For each ROI, five covariates were entered in the first step: age, IQ, experimental sequence, GMV in seed region, and GMV in the corresponding ROI. In the second step, EA and OXT were entered as main predictors. In the final step, an interaction $\mathrm{OXT} \times \mathrm{EA}$ was entered. All predictors were mean centered. Before model estimation, we calculated Mahalanobis distance to make sure that multivariate outliers were excluded. We further identified data points that exerted an excessive influence on the HMR model, using Cook's distances following each model estimation. Data points with a Cook's distance exceeding the sample specific cutoff (four/ sample size) were removed from the analysis, and the HMR model was re-estimated. We report results from the final HMR models free of outliers and influential data points. To examine an interaction effect, we tested whether successive regression steps significantly increased the variance explained by the model $\left(\Delta R^{2}\right)$.

\section{RESULTS}

\section{Psychological and Physiological Measures}

One subject was identified as an outlier because of a score of 21 on the SA subscale. SA has been associated with distinct patterns of stress reactivity compared with other ELS subtypes (Carpenter et al, 2009). For this reason, we excluded this subject and performed analyses on the data from 31 subjects (mean age $28.2 \pm 4.7$, mean IQ $112.5 \pm 15.5$, four left handed). Scores on the EN, EA, and PN subscales range from the none/low to severe ELS defined by previous normative data (Scher et al, 2001) (ie, - $1 \mathrm{SD} \leqslant$ the 50th percentile of the norm; +1 SD $\geqslant$ the 90th percentile of the norm; see Table 1). None of the subjects scored high on the subscale of PA and

Table I Demographic and Psychometric Measures

\begin{tabular}{lcc}
\hline & Mean (SD) & Range \\
\hline Age, years & $28.2(4.7)$ & $21-37$ \\
IQ & $112.5(15.5)$ & $92-145$ \\
& & \\
CTQ & & \\
Total score & $37.8(13.9)$ & $25-84$ \\
Emotional neglect & $10.5(4.9)$ & $5-23$ \\
Emotional abuse & $8.5(4.6)$ & $5-23$ \\
Physical neglect & $7.5(3.7)$ & $5-20$ \\
Physical abuse & $6.3(2.5)$ & $5-15$ \\
Sexual abuse & $5.1(0.3)$ & $5-6$
\end{tabular}

\section{NEO-FFI}

\begin{tabular}{lll} 
Neuroticism & $19.1(8.5)$ & $4-36$ \\
Extroversion & $29.3(6.5)$ & $12-40$ \\
Openness & $32.8(8.9)$ & $11-46$ \\
Agreeableness & $30.9(6.1)$ & $18-40$ \\
Conscientiousness & $30.1(7.4)$ & $11-40$ \\
& & \\
Rosenberg self-esteem scale & $40.0(5.9)$ & $23-50$ \\
& & \\
STAl & & $25-48$ \\
Trait anxiety & $36.5(7.0)$ & $26-49$ \\
State anxiety at baseline & $35.0(5.3)$ & $24-63$ \\
State anxiety after stress (PLA) & $39.8(8.0)$ & $22-64$ \\
State anxiety after stress (OXT) & $41.2(11.0)$ & \\
\hline
\end{tabular}

Abbreviations: CTQ, Childhood Trauma Questionnaire; OXT, oxytocin; PLA, placebo. 
SA subscale (ie, +1 SD not exceeding the 90th percentile of normative data; see Table 1).

Partial correlation analyses controlling for age and IQ showed that EA scores correlated positively with neuroticism $(r(23)=0.55, p<0.01)$ and trait anxiety $(r(23)=0.40$, $p<0.05)$ and negatively with self-esteem $(r(23)=-0.65$, $p<0.001)$. We observed higher state anxiety levels $(t(28)=$ $3.33, p<0.01)$ and salivary cortisol levels $(t(18)=2.55$, $p<0.05)$ after stress induction in the PLA session. EA correlated positively with state anxiety levels after stress induction regardless of the administration of OXT or PLA $\left(r_{\text {parital }}(50)=0.32, p<0.05\right)$. HMR revealed a significant OXT $\times$ EA interaction effect on the cortisol stress responses (beta $=0.430, t(35)=2.83, p<0.001)$. The full model with the interaction term was significant $(\mathrm{F}(6,35)=2.493, p<0.05)$, and adding the OXT $\times$ EA interaction as an additional predictor explained a significant proportion of variance in cortisol stress responses $\left(\Delta R^{2}=0.16\right.$, $\mathrm{F}$ Change $(1,35)=8.01$, $p<0.01)$. Post hoc analysis showed a negative correlation between EA scores and cortisol stress responses in the PLA session $\left(r_{\text {parital }}(14)=-0.43, p=0.098\right)$ and a positive correlation in the OXT session $\left(r_{\text {partial }}(18)=0.48, \quad p<0.05\right)$ (see Supplementary Figure S1).

\section{Effect of Psychosocial Stress on Amygdala FC}

To investigate the effect of psychosocial stress on amygdala coupling, we performed a PPI analysis for each subject's fMRI data in the placebo session. Whole-brain one-sample $T$-tests at group level revealed brain regions where activity was more positively coupled with that of bilateral amygdalae during psychosocial stress than during the control condition. Among these are the pregenual anterior cingulate cortex (pgACC), $\mathrm{mPFC}$, posterior cingulate cortex (PCC), left anterior insula, caudate, putamen, right thalamus, inferior parietal lobule (IPL), and bilateral cerebellum (see Supplementary Table S1,Supplementary Figure S2).

\section{Effect of EA on Stress-Induced Amygdala FC Shift}

Voxel-wise multiple regression in the placebo session further revealed regions where such stress-induced connectivity changes were modulated by EA. Specifically, as severity of EA increased, there was stronger stress-induced FC ascent between bilateral amygdalae and bilateral hippocampus extending to parahippocampal gyrus (PHG), bilateral fusiform gyrus (FFG), and cerebellum (see Table 2, Figure 1a).

In subsequent ROI-based multiple regression analysis controlling for age, IQ, experimental sequence, GMV, neuroticism, trait anxiety, and self-esteem, the main effect of EA on stress-induced FC enhancement remained significant for FC between the right amygdala seed and bilateral hippocampus extending to PHG, left FFG, and bilateral cerebellum (all beta's $>0.64$, all $p$ 's $<0.004$ ), as well as for FC between the left amygdala seed and right PHG, right FFG, and left precuneus (all beta's $>0.67$, all $p$ 's $<0.004)$. We also observed a significant association between neuroticism and stress-induced FC enhancement between the right amygdala seed and bilateral hippocampus/ PHG (all beta's $<-0.45$, all $p$ 's $<0.05$ ).
Table 2 Brain Regions in Which Stress-Induced Connectivity Changes were Predicted by Emotional Abuse

\begin{tabular}{lll}
\hline Region & \multicolumn{2}{c}{ Seed } \\
\hline & Left amygdala & Right amygdala \\
\hline Left Hipp/PHG & & $-30,-39,-12$ \\
& & $Z=3.9$ \\
Right Hipp & $39,-24,-15$ & $30,-33,-18$ \\
& $Z=3.29$ & $Z=4.28$ \\
Right PHG & $24,-33,-15$ & $36,-39,-15$ \\
& $Z=3.57$ & $Z=4.07$ \\
Left fusiform gyrus & & $-33,-39,-21$ \\
& & $Z=4.08$ \\
Right fusiform gyrus & $42,-42,-21$ & \\
Left precuneus & $Z=4.11$ & \\
Left cerebellum & $-15,-75,45$ & $-6,-57,-27$ \\
Right cerebellum & $Z=3.99$ & $Z=4.55$ \\
& $-6,-57,-30$ & $30,-48,-18$ \\
& $Z=4.18$ & $Z=3.7$ \\
\hline
\end{tabular}

Abbreviations: Hipp, hippocampus; PHG, parahippocampal gyrus. Results are reported at a threshold of cluster-level FWE-corrected $p<0.05$, cluster size $>50$ voxels. Coordinates are reported according to the $\mathrm{MNI}$ space.

\section{Joint Effect of EA and OXT on Stress-Induced Amygdala FC Shift}

To examine the main effect of OXT and the joint effect of OXT and EA on the stress-induced connectivity shift between the amygdala and hippocampus, a ROI was drawn in the right hippocampus where the effect of EA was observed. Stress-induced changes in amygdala-hippocampal FC were extracted for the placebo and OXT sessions separately. No significant main effect of OXT was found in the right hippocampus. HMR showed a significant OXT $\times$ EA interaction (beta $=-0.27, t(44)=-2.13, p<0.05)$. The full model with the interaction term was significant $\left(R^{2}=0.42\right.$, $\mathrm{F}(8,44)=3.90, p<0.01)$, and the OXT $\times \mathrm{EA}$ interaction explained a significant proportion of variance in amygdala-hippocampal FC $\left(\Delta R^{2}=0.06, \mathrm{~F}\right.$ Change $(1,44)=4.53$, $p<0.05)$. This ROI analysis further revealed that, in the placebo session, increased EA scores were associated with a positive shift in hippocampal-amygdala FC during psychosocial stress; while in the OXT session there was no significant correlation between EA scores and stress-induced FC changes between hippocampus and amygdala (see Figure $1 \mathrm{~b}$ ).

Similar OXT $\times$ EA interactions were also found in stressinduced FC changes from right amygdala to right PHG (beta $=-0.26, t(43)=-2.23, p<0.05$ ), from left amygdala to left fusiform gyrus (beta $=-0.34, t(44)=-3.27, p<0.01$ ), as well as from bilateral amygdalae to left cerebellum (for 1 Amg, beta $=-0.41, t(44)=-2.88, p<0.01$; for $\mathrm{r}$ Amg, beta $=-0.47, t(45)=-3.97, p<0.001$; for detailed results of the HMR models, please see Supplementary Table S2).

Correlation analyses with the physiological data showed that stress-induced FC changes between the right amygdala 

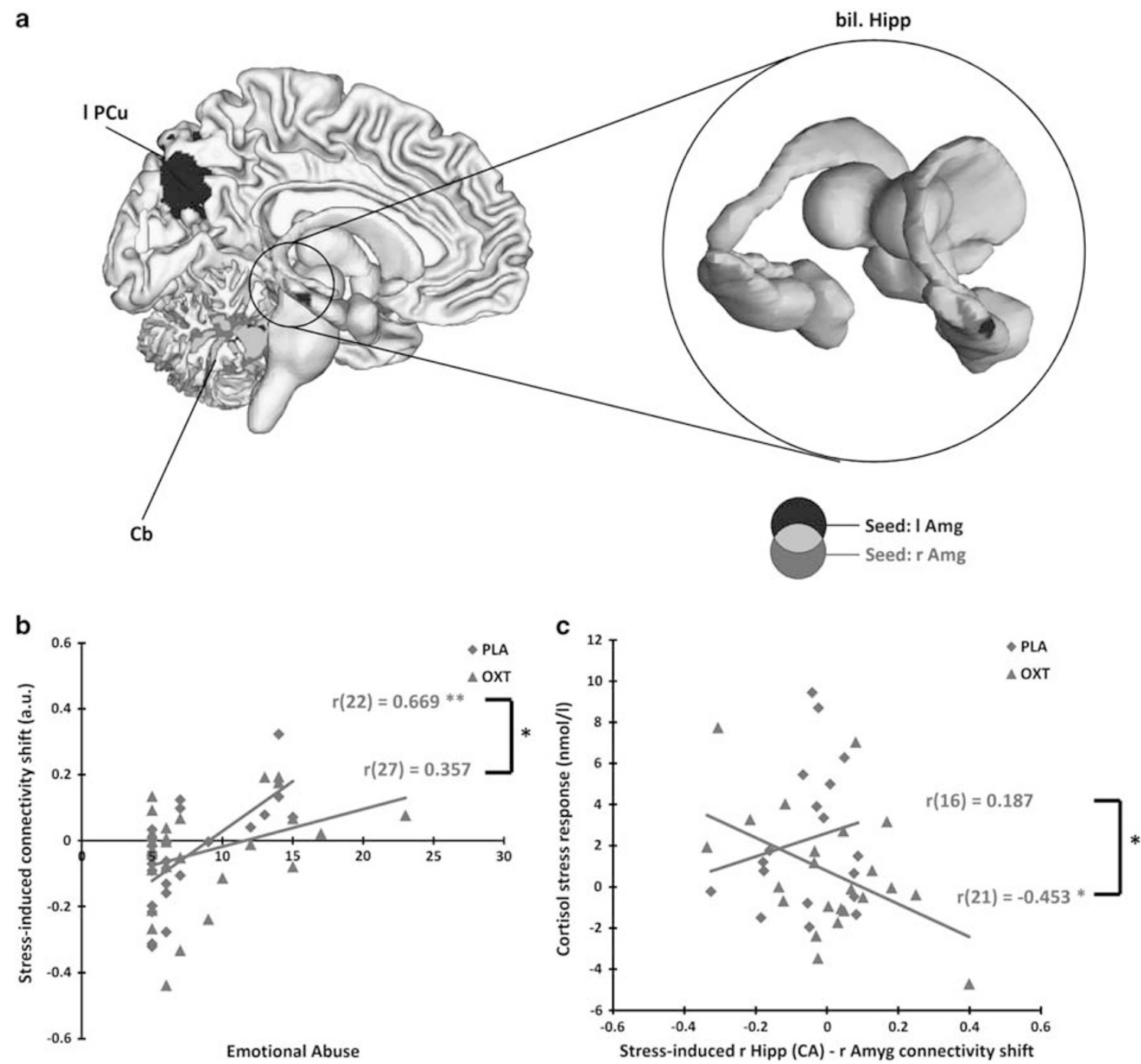

Figure I (a) Voxel-wise multiple regression analysis of the placebo session showed that emotional abuse positively predicted stress-induced connectivity enhancement between the amygdala and the illustrated regions (cluster-level FWE-corrected $p<0.05$, cluster size $>50$ voxels). (b) Results of the hierarchical multiple regression estimating the joint effect of oxytocin and emotional abuse on stress-induced connectivity shift between right amygdala and right hippocampus. (c) Cortisol stress response correlated negatively with the stress-induced connectivity shift between right amygdala and right hippocampus Cornu Ammonis (CA) areas during the oxytocin session but not the placebo session. Amg, amygdala; Cb, cerebellum; Hipp, hippocampus; OXT, oxytocin; PCu, precuneus; PLA, placebo.

and right hippocampus correlated negatively with cortisol stress responses during the OXT session $(r(21)=-0.453$, $p<0.05)$ but not during the placebo session $(r(16)=0.187$, $p>0.45$; Fischer's $Z=1.98, p<0.05$; see Figure 1c). Similar correlation pattern was also found in stress-induced FC changes between the right amygdala and right PHG (for OXT session, $r(20)=-0.697, p<0.001$; for PLA session, $r$ $(15)=0.137, p>0.60$; Fischer's $Z=2.83, p<0.001)$.

\section{DISCUSSION}

The first aim of the present study was to examine the impact of EA on stress-induced changes in amygdala-centered FC. Consistent with previous findings (Hermans et al, 2011), psychosocial stress induced increased FC strength between the amygdala and distributed stress-related cortical-subcortical regions when compared with the control condition.
Among these are regions associated with salience processing and autonomic-neuroendocrine control (ie, $\mathrm{mPFC} / \mathrm{pgACC}$, insula, caudate, putamen, and thalamus), as well as attentional (re)orientation and executive control (ie, MCC, PCC/precuneus, IPL, and cerebellum). Moreover, early life experience of EA positively predicted stress-induced FC enhancement between bilateral amygdalae and the right hippocampus/PHG. This linear association remained significant after controlling for individual differences in age, IQ, experimental sequence, GMV of corresponding areas, neuroticism, self-esteem, and trait anxiety. ELS has previously been associated with limbic hyper-responsiveness to negative facial cues during early stage of emotion processing (Dannlowski et al, 2013). Impaired structural connectivity within the limbic-prefrontal circuit has also been observed in subjects who have experienced ELS (Hart and Rubia, 2012). Recently, ELS has also been associated with decreased amygdala-mPFC and hippocampal-mPFC FC during 
resting state (Burghy et al, 2012; Fan et al, 2014; Herringa et al, 2013), suggesting ELS-associated aberration in the traitlike functional integrity of the limbic-prefrontal circuit. Adding to this evidence, our finding highlights the association between EA and transient task-evoked changes in amygdala-hippocampal FC in response to acute psychosocial stress.

The amygdala-hippocampal pathway is crucially involved in the regulation of HPA axis (Herman et al, 2005). Kiem et al (2013) reported that stronger amygdala-hippocampal rs-FC is associated with dampened cortisol responses to Dex/ $\mathrm{CRH}$ test, which indicates decreased efficiency of the HPA axis to re-establish homeostasis after perturbation. Vaisvaser et al (2013) found prolonged increases in amygdalahippocampal FC even $2 \mathrm{~h}$ after psychosocial stress, which was associated with blunted cortisol stress responses. These findings associate increased amygdala-hippocampal coupling with dysregulated HPA stress reactivity, especially aberrant glucocorticoid negative feedback. Substantial evidence associates ELS with dysregulated cortisol stress responses and HPA reactivity (Strüber et al, 2014). It has recently been found that ELS can induce epigenetic modification in the hippocampus, which is related to deficiency in HPA regulation via glucocorticoid negative feedback (Klengel et al, 2013). Consistent with these previous findings, the ELS-associated enhancement in stress-induced amygdala-hippocampal FC may mediate the impact of ELS on neuroendocrine control. The stress regulation circuit and the neural circuit for learning and memory largely overlap in the amygdala and hippocampus and enhanced connectivity between these two regions has an important role in both encoding and consolidation of emotional memory (RichterLevin and Akirav, 2000). Acute stress alters hippocampal activity during encoding and affects memory performance (Henckens et al, 2009). The ELS-associated enhancement in stress-induced amygdala-hippocampal FC changes may therefore indicate an impact of ELS on memory during stress.

It should be noted that although we observed stressinduced changes in FC between bilateral amygdalae and the $\mathrm{mPFC} / \mathrm{pgACC}$, these were not influenced by EA even at ROI level (see Supplementary Material). At a first glance, this seems inconsistent with previous findings that ELS is associated with decreased amygdala-prefrontal FC during resting state (Burghy et al, 2012; Fan et al, 2014; Herringa et al, 2013). However, it is important to emphasize that task-evoked FC changes reflect transient changes in brain network coupling in response to specific task demands. The stress-induced FC change assesses reactivity of the stress regulation circuit in response to a transient challenge, which is complimentary to the trait-like measurement of task-independent FC during resting state. Cousijn et al (2012) recently showed that stress induction modulates both tonic and phasic activity in the amygdala but only phasic activity in the hippocampus. The ELS-associated enhancement in stress-induced amygdala-hippocampal FC is likely to reflect the impact of ELS on phasic FC changes in the amygdala-hippocampal pathway in response to acute stress, while rs-FC changes between amygdala and the $\mathrm{mPFC} / \mathrm{pgACC}$ may reflect the influence of ELS on more tonic network properties in the amygdala-prefrontal pathway.
Furthermore, we found a joint effect of OXT and EA on the stress-induced changes in amygdala-hippocampal FC. Specifically, OXT induced a positive shift in stress-induced FC changes in subjects with lower EA scores but a negative shift in subjects with higher EA scores. The changes in amygdala-hippocampal FC in the OXT session were negatively associated with cortisol stress responses. This concurred with the physiological measure that cortisol stress responses in the OXT condition were reduced in subjects with lower EA scores but were increased in subjects with higher EA scores. Previous findings demonstrated that OXT modulates HPA stress responses, and this stress-attenuating effect is mediated via the inhibition of central-medial amygdala (Huber et al, 2005). It has further been reported that the stress-attenuating effect of OXT is altered in subjects who have experienced ELS (Bakermans-Kranenburg and van IJzendoorn, 2013). Our recent study revealed that alteration in stress-induced limbic deactivation is involved in the ELSdependent sensitivity to OXT (Grimm et al, 2014). In addition to these findings, we demonstrated ELS-dependent OXT effects on stress-induced changes in amygdala-hippocampal FC. Considering the inverse association between amygdala-hippocampal FC and cortisol stress responses, our finding is in concordance with the altered stress-attenuating effect of OXT in subjects who have experienced ELS. To our knowledge, this is the first study associating stress-induced phasic FC changes in the amygdala-hippocampal pathway with ELS-dependent sensitivity to OXT.

It is worth noticing that the oxytocinergic system itself is highly sensitive to the impact of ELS (Heim et al, 2009; Hill et al, 2014). From an early developmental stage, the endogenous secretion of OXT and the expression of OXT receptors (especially in the amygdala) are modulated by the amount and quality of maternal affectionate behavior (Hill et al, 2014). Other than the ELS-associated enhancement of stress-induced amygdala-hippocampal FC, ELS-associated dysfunction in the oxytocinergic system itself may also be an intermediate factor for altered OXT effects.

It has been proposed that OXT is advantageous for the treatment of stress-related psychiatric disorders such as social phobia due to its stress-attenuating effect (see review in Bakermans-Kranenburg and van IJzendoorn, 2013). However, recent evidence reveals that OXT is not always beneficial depending on various modulating factors, eg, the personal history of ELS (Bakermans-Kranenburg and van IJzendoorn, 2013). Regarding the neural mechanism of the ELS-dependent sensitivity to OXT, we have previously shown differential OXT effects on pgACC stress responses depending on resting-state amygdala-pgACC FC before stress induction (Fan et al, 2014). Our findings in the present study provide further support for the differential effects of OXT on task-evoked amygdala-hippocampus FC during stress. As both trait-like basal rs-FC and stress-induced phasic FC changes within the limbic-prefrontal circuit are modulated by ELS, these findings emphasize the necessity to consider the tonic basal state and phasic functional reactivity of the limbic-prefrontal circuit when assessing the stressattenuating effect of OXT.

Although our study provides new insights regarding the joint effect of EA and OXT on stress-induced changes in amygdala-hippocampal FC, several limitations need to be considered. The CTQ assesses ELS based on retrospective 
self-report and is hence susceptible to subjectivity bias in perception of early environment and in reconstructive memory. We did not include any 'objective' measure of ELS (eg, medical records). However, it was mainly on the EA and EN subscale that our subjects reported moderate-tosevere ELS (Bernstein and Fink, 1998; Scher et al, 2001). Such information on emotional maltreatment is unlikely to be reflected in medical records. In addition, many subjects were not in contact with their families, so that family interviews would have been difficult to arrange. As the perception and recall of ELS are influenced by various factors, including personality traits and coping skills, it is important to acknowledge the subjectivity in our measurement of EA when interpreting the findings. Previous studies showed differential effects of ELS on stress reactivity in a gender- and age-specific manner, depending on the specific type and severity of ELS, as well as the presence/absence of concurrent stress and psychiatric symptoms (Heim and Binder, 2012; Strüber et al, 2014). In the present study, we recruited only healthy young male subjects and used EA as the main predictor in our multiple regression models. Although this helps to delineate the effect of EA from that of other subtypes of ELS and from the influence of psychiatric symptoms, one should be cautious when generalizing our findings to female samples or subjects with concurrent psychiatric symptoms. As we did not obtain any longitudinal data, it is not known whether the subjects will develop psychiatric disorders later in life. Future studies are needed to differentiate individuals vulnerable and resilient to the detrimental influence of ELS. It is also important to point out that the pharmacokinetics of OXT and its effect at molecular biology level remain unknown. The exact effect of OXT on limbic-prefrontal network may be much more complicated on a micro-circuity level. For example, it has been suggested that OXT inhibits activity in the amygdala via its interaction with the GABAergic and serotonergic system within different nuclei of the amygdala complex (Strüber et al, 2014). Although our findings associate the limbic-prefrontal circuit with the stress-attenuating effect of OXT on a macro-network scale, further cellular biological research is needed to elucidate the effect of OXT on a micro-circuitry level.

In conclusion, we found that EA is positively associated with enhanced stress-induced FC strength between the amygdala and hippocampus, whereas this association is not significant after the administration of intranasal OXT. Stressinduced amygdala-hippocampal FC is crucial in the neural mechanism underlying the impact of ELS on stress reactivity and may also be involved in the ELS-dependent stressattenuating effect of OXT.

\section{FUNDING AND DISCLOSURE}

This work was supported by the German Research Foundation (DFG, Cluster of Excellence 'Languages of Emotion'). The authors declare no conflict of interest.

\section{ACKNOWLEDGMENTS}

We thank the team of Dahlem Institute for Neuroimaging of Emotion for their technical support, Jue Wang for helpful suggestions with the data analysis, and Anne Weigand and Matti Gärtner for comments on the manuscript.

\section{REFERENCES}

Bakermans-Kranenburg MJ, van IJzendoorn MH (2013). Sniffing around oxytocin: review and meta-analyses of trials in healthy and clinical groups with implications for pharmacotherapy. Transl Psychiatry 3: e258.

Bernstein DP, Fink L (1998). Childhood Trauma Questionnaire: A retrospective self-report manual. The Psychological Corporation: San Antonio, TX, USASan Antonio, TX, USA.

Burghy CA, Stodola DE, Ruttle PL, Molloy EK, Armstrong JM, Oler JA et al (2012). Developmental pathways to amygdalaprefrontal function and internalizing symptoms in adolescence. Nat Neurosci 15: 1736-1741.

Carpenter LL, Tyrka AR, Ross NS, Khoury L, Anderson GM, Price LH (2009). Effect of childhood emotional abuse and age on cortisol responsivity in adulthood. Biol Psychiatry 66: 69-75.

Costa PT, McCrae RR (1985). The NEO Personality Inventory manual. Psychological Assessment Resources: Odessa, FL, USA.

Cousijn H, Rijpkema M, Qin S, van Wingen GA, Fernandez G (2012). Phasic deactivation of the medial temporal lobe enables working memory processing under stress. Neuroimage 59: 1161-1167.

Dannlowski U, Kugel H, Huber F, Stuhrmann A, Redlich R, Grotegerd D et al (2013). Childhood maltreatment is associated with an automatic negative emotion processing bias in the amygdala. Hum Brain Mapp 34: 2899-2909.

Dressendorfer RA, Kirschbaum C, Rohde W, Stahl F, Strasburger CJ (1992). Synthesis of a cortisol-biotin conjugate and evaluation as a tracer in an immunoassay for salivary cortisol measurement. J Steroid Biochem Mol Biol 43: 683-692.

Fan Y, Herrera-Melendez AL, Pestke K, Feeser M, Aust S, Otte C et al (2014). Early life stress modulates amygdala-prefrontal functional connectivity: Implications for oxytocin effects. Hum Brain Mapp 35: 5328-5339.

Freese JL, Amaral DG (2009)Neuroanatomy of the primate amygdalaIn:Whalen PJ, Phelps EA(eds). The Human Amygdala. The Guilford Press: New York, NY, USA, pp 3-42.

Friston KJ, Buechel C, Fink GR, Morris J, Rolls E, Dolan RJ (1997). Psychophysiological and modulatory interactions in neuroimaging. Neuroimage 6: 218-229.

Fuge P, Aust S, Fan Y, Weigand A, Gartner M, Feeser M et al (2014). Interaction of early life stress and corticotropin-releasing hormone receptor gene: effects on working memory. Biol Psychiatry 76: 888-894.

Gamer M, Zurowski B, Buchel C (2010). Different amygdala subregions mediate valence-related and attentional effects of oxytocin in humans. Proc Natl Acad Sci USA 107: 9400-9405.

Gee DG, Gabard-Durnam LJ, Flannery J, Goff B, Humphreys KL, Telzer EH et al (2013). Early developmental emergence of human amygdala-prefrontal connectivity after maternal deprivation. Proc Natl Acad Sci USA 110: 15638-15643.

Grimm S, Pestke K, Feeser M, Aust S, Weigand A, Wang J et al (2014). Early life stress modulates oxytocin effects on limbic system during acute psychosocial stress. Soc Cogn Affect Neurosci 9: $1828-1835$.

Hart H, Rubia K (2012). Neuroimaging of child abuse: a critical review. Front Hum Neurosci 6: 52.

Heim C, Binder EB (2012). Current research trends in early life stress and depression: review of human studies on sensitive periods, gene-environment interactions, and epigenetics. Exp Neurol 233: 102-111.

Heim C, Young LJ, Newport DJ, Mletzko T, Miller AH, Nemeroff CB (2009). Lower CSF oxytocin concentrations in women with a history of childhood abuse. Mol Psychiatry 14: 954-958. 
Henckens MJ, Hermans EJ, Pu Z, Joels M, Fernandez G (2009). Stressed memories: how acute stress affects memory formation in humans. J Neurosci 29: 10111-10119.

Herman JP, Ostrander MM, Mueller NK, Figueiredo H (2005). Limbic system mechanisms of stress regulation: hypothalamopituitary-adrenocortical axis. Prog Neuropsychopharmacol Biol Psychiatry 29: 1201-1213.

Hermans EJ, van Marle HJ, Ossewaarde L, Henckens MJ, Qin S, van Kesteren MT et al (2011). Stress-related noradrenergic activity prompts large-scale neural network reconfiguration. Science 334: 1151-1153.

Herringa RJ, Birn RM, Ruttle PL, Burghy CA, Stodola DE, Davidson RJ et al (2013). Childhood maltreatment is associated with altered fear circuitry and increased internalizing symptoms by late adolescence. Proc Natl Acad Sci USA 110: 19119-19124.

Hill KT, Warren M, Roth TL (2014). The influence of infantcaregiver experiences on amygdala Bdnf, OXTr, and NPY expression in developing and adult male and female rats. Behav Brain Res 272C: 175-180.

Holz NE, Buchmann AF, Boecker R, Blomeyer D, Baumeister S, Wolf I et al (2015). Role of FKBP5 in emotion processing: results on amygdala activity, connectivity and volume. Brain Struct Funct 200: 1355-1368.

Huber D, Veinante P, Stoop R (2005). Vasopressin and oxytocin excite distinct neuronal populations in the central amygdala. Science 308: 245-248.

Keyes KM, Eaton NR, Krueger RF, McLaughlin KA, Wall MM, Grant BF et al (2012). Childhood maltreatment and the structure of common psychiatric disorders. Br J Psychiatry 200: 107-115.

Kiem SA, Andrade KC, Spoormaker VI, Holsboer F, Czisch M, Samann PG (2013). Resting state functional MRI connectivity predicts hypothalamus-pituitary-axis status in healthy males. Psychoneuroendocrinology 38: 1338-1348.

Klengel T, Mehta D, Anacker C, Rex-Haffner M, Pruessner JC, Pariante CM et al (2013). Allele-specific FKBP5 DNA demethylation mediates gene-childhood trauma interactions. Nat Neurosci 16: 33-41.

Labuschagne I, Phan KL, Wood A, Angstadt M, Chua P, Heinrichs $\mathrm{M}$ et al (2010). Oxytocin attenuates amygdala reactivity to fear in generalized social anxiety disorder. Neuropsychopharmacology 35: 2403-2413.

LeDoux JE (2000). Emotion circuits in the brain. Annu Rev Neurosci 23: $155-184$

Lehrl S, Triebig G, Fischer B (1995). Multiple choice vocabulary test MWT as a valid and short test to estimate premorbid intelligence. Acta Neurol Scand 91: 335-345.

McEwen BS (2003). Mood disorders and allostatic load. Biol Psychiatry 54: 200-207.

McLaren DG, Ries ML, Xu G, Johnson SC (2012). A generalized form of context-dependent psychophysiological interactions (gPPI): a comparison to standard approaches. Neuroimage 61: 1277-1286.

Nelson HE, O'Connell A (1978). Dementia: the estimation of premorbid intelligence levels using the New Adult Reading Test. Cortex 14: 234-244.

Neumann ID, Wigger A, Torner L, Holsboer F, Landgraf R (2000). Brain oxytocin inhibits basal and stress-induced activity of the hypothalamo-pituitary-adrenal axis in male and female rats: partial action within the paraventricular nucleus. J Neuroendocrinol 12: 235-243.

Pechtel P, Pizzagalli DA (2011). Effects of early life stress on cognitive and affective function: an integrated review of human literature. Psychopharmacology (Berl) 214: 55-70.

Pruessner JC, Dedovic K, Khalili-Mahani N, Engert V, Pruessner M, Buss C et al (2008). Deactivation of the limbic system during acute psychosocial stress: evidence from positron emission tomography and functional magnetic resonance imaging studies. Biol Psychiatry 63: 234-240.

Richter-Levin G, Akirav I (2000). Amygdala-hippocampus dynamic interaction in relation to memory. Mol Neurobiol 22: 11-20.

Riem MM, van IMH, Tops $M$, Boksem MA, Rombouts SA, Bakermans-Kranenburg MJ (2012). No laughing matter: intranasal oxytocin administration changes functional brain connectivity during exposure to infant laughter. Neuropsychopharmacology 37: 1257-1266.

Rosenberg M (1965). Society and the Adolescent Self-Image. Princeton University Press: Princeton, NJ, USAPrinceton, NJ, USA.

Scher CD, Stein MB, Asmundson GJ, McCreary DR, Forde DR (2001). The childhood trauma questionnaire in a community sample: psychometric properties and normative data. J Trauma Stress 14: 843-857.

Schmidt KH, Metzler P (1992). Wortschatztest (WST). Beltz Test GmbH: Weinheim, GermanyWeinheim, Germany.

Spielberger CD, Sydeman SJ (1994). State-Trait Anxiety Inventory and State-Trait Anger Expression Inventory. Lawrence Erlbaum Associates: Hillsdale, NJ, USA.

Strüber N, Strüber D, Roth G (2014). Impact of early adversity on glucocorticoid regulation and later mental disorders. Neurosci Biobehav Rev 38: 17-37.

Teicher MH, Anderson CM, Polcari A (2012). Childhood maltreatment is associated with reduced volume in the hippocampal subfields CA3, dentate gyrus, and subiculum. Proc Natl Acad Sci USA 109: E563-E572.

Tzourio-Mazoyer N, Landeau B, Papathanassiou D, Crivello F, Etard O, Delcroix N et al (2002). Automated anatomical labeling of activations in SPM using a macroscopic anatomical parcellation of the MNI MRI single-subject brain. Neuroimage 15: 273-289.

Vaisvaser S, Lin T, Admon R, Podlipsky I, Greenman Y, Stern N et al (2013). Neural traces of stress: cortisol related sustained enhancement of amygdala-hippocampal functional connectivity. Front Hum Neurosci 7: 313.

Veer IM, Oei NY, Spinhoven P, van Buchem MA, Elzinga BM, Rombouts SA (2011). Beyond acute social stress: increased functional connectivity between amygdala and cortical midline structures. Neuroimage 57: 1534-1541.

Wager TD, Barrett LF, Bliss-Moreau E, Lindquist KA, Duncan S, Kober $\mathrm{H}$ et al (2012)The neuroimaging of emotionIn:Lewis $\mathrm{M}$, Haviland-Jones JM, Barrett LF(eds). Handbook of Emotions. The Guilford Press: New York, NY, USA, pp 249-271.

Wittchen HU, Wunderlich U, Gruschwitz S, Zaudig M (1997). SKID-I. Strukturiertes Klinisches Interview für DSM-IV. Achse I: Psychische Störungen. Hogrefe: Göttingen, GermanyGöttingen, Germany.

Supplementary Information accompanies the paper on the Neuropsychopharmacology website (http://www.nature.com/npp) 